\title{
Hyers-Ulam-Rassias Stability for the Heat Equation
}

\author{
Maher Nazmi Qarawani \\ Department of Mathematics, AlQuds Open University, Salfit, Palestine \\ Email: mkerawani@qou.edu
}

Received April 26, 2013; revised May 27, 2013; accepted June 5, 2013

Copyright ( 2013 Maher Nazmi Qarawani. This is an open access article distributed under the Creative Commons Attribution License, which permits unrestricted use, distribution, and reproduction in any medium, provided the original work is properly cited.

\begin{abstract}
In this paper we apply the Fourier transform to prove the Hyers-Ulam-Rassias stability for one dimensional heat equation on an infinite rod. Further, the paper investigates the stability of heat equation in $\mathbb{R}^{n}$ with initial condition, in the sense of Hyers-Ulam-Rassias. We have also used Laplace transform to establish the modified Hyers-Ulam-Rassias stability of initial-boundary value problem for heat equation on a finite rod. Some illustrative examples are given.
\end{abstract}

Keywords: Hyers-Ulam-Rassias Stability; Heat Equation; Fourier Transform; Laplace Transform

\section{Introduction and Preliminaries}

The study of stability problems for various functional equations originated from a famous talk given by Ulam in 1940. In the talk, Ulam discussed a problem concerning the stability of homomorphisms. A significant breakthrough came in 1941, when Hyers [1] gave a partial solution to Ulam's problem. Afterthen and during the last two decades a great number of papers have been extensively published concerning the various generalizations of Hyers result (see [2-10]).

Alsina and Ger [11] were the first mathematicians who investigated the Hyers-Ulam stability of the differential equation $g^{\prime}=g$. They proved that if a differentiable function $y: I \rightarrow R$ satisfies $\left|y^{\prime}-y\right| \leq \varepsilon$ for all $t \in I$, then there exists a differentiable function $g: I \rightarrow R$ satisfying $g^{\prime}(t)=g(t)$ for any $t \in I$ such that $|g-y| \leq 3 \varepsilon$, for all $t \in I$. This result of alsina and Ger has been generalized by Takahasi et al. [12] to the case of the complex Banach space valued differential equation $y^{\prime}=\lambda y$.

Furthermore, the results of Hyers-Ulam stability of differential equations of first order were also generalized by Miura et al. [13], Jung [14] and Wang et al. [15].

$\mathrm{Li}$ [16] established the stability of linear differential equation of second order in the sense of the Hyers and Ulam $y^{\prime \prime}=\lambda y$. Li and Shen [17] proved the stability of nonhomogeneous linear differential equation of second order in the sense of the Hyers and Ulam

$y^{\prime \prime}+p(x) y^{\prime}+q(x) y+r(x)=0$, while Gavruta et al. [18] proved the Hyers-Ulam stability of the equation $y^{\prime \prime}+\beta(x) y=0$ with boundary and initial conditions.
Jung [19] proved the Hyers-Ulam stability of first-order linear partial differential equations. Gordji et al. [20] generalized Jung's result to first order and second order Nonlinear partial differential equations. Lungu and Craciun [21] established results on the Ulam-Hyers stability and the generalized Ulam-HyersRassias stability of nonlinear hyperbolic partial differential equations.

In this paper we consider the Hyers-Ulam-Rassias stability of the heat equation

$$
\frac{\partial u}{\partial t}=a^{2} \frac{\partial^{2} u}{\partial x^{2}} \quad 0<t \leq T<\infty,-\infty<x<\infty
$$

with the initial condition

$$
u(x, 0)=\mu(x)
$$

where $\mu(x) \in C(-\infty, \infty)$, and

$$
u(x, t) \in C_{1}^{2}(\mathbb{R} \times(0, \infty)) .
$$

We also use a similar argument to establish the HyersUlam-Rassias for the heat equation in higher dimension

$$
u_{t}=a^{2} \Delta u \quad 0<t \leq T<\infty, x \in \mathbb{R}^{n}
$$

with the initial condition

$$
u(x, 0)=\mu(x)
$$

where $\Delta u=\sum_{i=1}^{n} u_{x_{i} x_{i}}$.

Moreover we have proved theorems on Hyers-UlamRassias-Gavruta stability for the heat equation in a finite rod.

Definition 1 We will say that the Equation (1) has the 
Hyers-Ulam-Rassias stability with respect to $\varphi>0$, if there exists $K>0$ such that for each $\varepsilon>0$ and for each solution $u(x, t) \in C_{1}^{2}\left(\mathbb{R}^{n} \times(0, \infty)\right)$ of the inequality

$$
\left|u_{t}-a^{2} \Delta u\right| \leq \varepsilon \varphi(x, t)
$$

with the initial condition (2), then there exists a solution $w(x, t) \in C_{1}^{2}\left(\mathbb{R}^{n} \times(0, \infty)\right)$ of the Equation (1), such that

$$
\begin{gathered}
|u(x, t)-w(x, t)| \leq K \varepsilon \varphi(x, t), \\
\forall(x, t) \in \mathbb{R}^{n} \times(0, \infty),
\end{gathered}
$$

where $K$ is a constant that does not depend on $\varepsilon$ nor on $u(x, t)$, and $\varphi(x, t) \in C\left(\mathbb{R}^{n} \times(0, \infty)\right)$.

Definition 2 We will say that the equation (1) has the Hyers-Ulam-Rassias-Gavruta (HURG) stability with respect to $\varphi>0$, if there exists $K>0$ such that for each $\varepsilon>0$ and for each solution $u(x, t) \in C_{1}^{2}\left(\mathbb{R}^{n} \times(0, \infty)\right)$ of the inequality

$$
\left|u_{t}-a^{2} \Delta u\right| \leq \varepsilon \varphi(x, t)
$$

with the initial condition (2), then there exists a solution $w(x, t) \in C_{1}^{2}\left(\mathbb{R}^{n} \times(0, \infty)\right)$ of the Equation (1), such that

$$
\begin{gathered}
|u(x, t)-w(x, t)| \leq K \varepsilon \varphi(x, t), \\
\forall(x, t) \in \mathbb{R}^{n} \times(0, \infty),
\end{gathered}
$$

where $K$ is a constant that does not depend on $\varepsilon$ nor on $u(x, t)$, and $\varphi(x, t) \in C\left(\mathbb{R}^{n} \times(0, \infty)\right)$.

Definition 3 We will say that the solution of the initial value problem (1), (2) has the Hyers-Ulam-Rassias asymptotic stability with respect to $\varphi>0$, if it is stable in the sense of Hyers and Ulam with respect to $\varphi$, and

$$
\lim _{t \rightarrow \infty}(u(x, t)-w(x, t))=0
$$

Definition 4 Assume the functions $f(x)$ and $g(x)$ defined on $x \in \mathbb{R}^{n}$ are continuously differentiable and absolutely integrable, then the Fourier transform of $f(x)$ is defined as

$$
\mathcal{F}[f]=\frac{1}{(2 \pi)^{n / 2}} \int_{\mathbb{R}^{n}} f(x) \mathrm{e}^{-i \xi x} \mathrm{~d} x=F(\xi)
$$

and the inverse Fourier transform of $G(\xi), \xi \in \mathbb{R}^{n}$ is

$$
\mathcal{F}^{-1}[g]=\frac{1}{(2 \pi)^{n / 2}} \int_{\mathbb{R}^{n}} G(\xi) \mathrm{e}^{i \xi x} \mathrm{~d} \xi=g(x)
$$

\section{Example 1 Let}

$$
f(x)=\mathrm{e}^{-\beta|x|^{2}}, x \in \mathbb{R}^{n}, \beta>0
$$

We find the Fourier transform of the function. Since

$$
\begin{aligned}
f(x) & =\mathrm{e}^{-\beta|x|^{2}}=\mathrm{e}^{-\beta\left(x_{1}^{2}+\cdots+x_{n}^{2}\right)}=\mathrm{e}^{-\beta x_{1}^{2}} \cdots \mathrm{e}^{-\beta x_{n}^{2}} \\
& =h\left(x_{1}\right) \cdot h\left(x_{2}\right) \cdots h\left(x_{n}\right)
\end{aligned}
$$

Then

$$
h\left(x_{k}\right)=\mathrm{e}^{-\beta x_{k}^{2}}, k=1, \cdots, n
$$

and by defintion 4 we have

$$
F(\xi)=\prod_{k=1}^{n} H\left(\xi_{k}\right)
$$

where

$$
H\left(\xi_{k}\right)=\frac{1}{(2 \pi)^{1 / 2}} \int_{-\infty}^{\infty} \mathrm{e}^{-\beta x_{k}^{2}} \mathrm{e}^{-i \xi_{k} x_{k}} \mathrm{~d} x_{k}
$$

Differentiating $H\left(\xi_{k}\right)$ with respect to $\xi_{k}$, we get

$$
H^{\prime}\left(\xi_{k}\right)=\frac{1}{(2 \pi)^{n / 2}} \int_{-\infty}^{\infty} \mathrm{e}^{-\beta x_{k}^{2}} \mathrm{e}^{-i \xi_{k} x_{k}}\left(-i x_{k}\right) \mathrm{d} x_{k}
$$

Integrating by parts gives

$$
H^{\prime}\left(\xi_{k}\right)=\frac{\xi_{k}}{2 \beta} H\left(\xi_{k}\right)
$$

Hence

$$
H\left(\xi_{k}\right)=C \mathrm{e}^{-\xi_{k}^{2} / 4 \beta}
$$

Putting $\xi_{k}=0$ gives $C=H(0)$, and from (8) one has

$$
H(0)=\frac{1}{(2 \pi)^{1 / 2}} \int_{-\infty}^{\infty} \mathrm{e}^{-\beta x_{k}^{2}} \mathrm{~d} x_{k}
$$

Using that $\int_{-\infty}^{\infty} \mathrm{e}^{-\beta z^{2}} \mathrm{~d} z=\sqrt{\frac{\pi}{\beta}}$, we have

$$
\begin{gathered}
H(0)=\frac{1}{(2 \beta)^{1 / 2}} \\
H\left(\xi_{k}\right)=\frac{1}{(2 \beta)^{1 / 2}} \mathrm{e}^{-\xi_{k}^{2} / 4 \beta}
\end{gathered}
$$

Therefore, from (7), (9) we obtain

$$
F(\xi)=\frac{1}{(2 \beta)^{n / 2}} \mathrm{e}^{-|\xi|^{2} / 4 \beta}
$$

Theorem 1 (See Evans [22]) Assume that $f(x)$ and $g(x)$ are continuously differentiable and absolutely integrable on $\mathbb{R}^{n}$. Then

1) for each $\alpha$ such that $D^{\alpha} f \in L\left(\mathbb{R}^{n}\right)$, $\mathcal{F}\left[D^{\alpha} f\right]=(i \xi)^{n} \mathcal{F}[f]$.

2) $\mathcal{F}[f * g]=(2 \pi)^{\frac{n}{2}} \mathcal{F}[f] \mathcal{F}[g]$, where $f * g=\int_{\mathbb{R}^{n}} f(y) g(x-y)$ dy is the convolution of $f(x)$ 
and $g(x)$.

\section{On Hyers-Ulam-Rassias Stability for Heat Equation on an Infinite Rod}

Theorem 2 If $u(x, t) \in C_{1}^{2}(\mathbb{R} \times(0, T])$ then the initial value problem (1), (2) is stable in the sense of HyersUlam-Rassias.

Proof. Let $\varepsilon>0$ and $u(x, t)$ be an approximate solution of the initial value problem (1), (2). We will show that there exists a function $w(x, t) \in C_{1}^{2}(\mathbb{R} \times(0, T])$ satisfying the Equation (1) and the initial condition (2) such that

$$
|u(x, t)-w(x, t)| \leq K \varepsilon \varphi(x, t)
$$

If we take $\varphi(x, t)=\frac{1}{2 a \sqrt{(t+1)}} \mathrm{e}^{-\frac{1}{4 a^{2}} \frac{x^{2}}{t+1}}$ then from inequality (5), we have

$$
\begin{aligned}
\frac{-\varepsilon}{2 a \sqrt{(t+1)}} \mathrm{e}^{-\frac{1}{4 a^{2}} \frac{x^{2}}{t+1}} & \leq \frac{\partial u}{\partial t}-a^{2} \frac{\partial^{2} u}{\partial x^{2}} \\
& \leq \frac{\varepsilon}{2 a \sqrt{(t+1)}} \mathrm{e}^{-\frac{1}{4 a^{2}} \frac{x^{2}}{t+1}}
\end{aligned}
$$

Applying Fourier Transform to inequality (10), we get

$$
-\varepsilon \mathrm{e}^{-a^{2} \xi^{2}(t+1)} \leq \frac{\mathrm{d} v(\xi, t)}{\mathrm{d} t}+a^{2} \xi^{2} v(\xi, t) \leq \varepsilon \mathrm{e}^{-a^{2} \xi^{2}(t+1)}
$$

Or, equivalently

$$
\begin{aligned}
-\varepsilon T \mathrm{e}^{-a^{2} \xi^{2}} & \leq \mathrm{e}^{a^{2} \xi^{2} t} \frac{\mathrm{d} v(\xi, t)}{\mathrm{d} t}+a^{2} \xi^{2} v(\xi, t) \mathrm{e}^{a^{2} \xi^{2} t} \\
& \leq \varepsilon T \mathrm{e}^{-a^{2} \xi^{2}}
\end{aligned}
$$

Integrating the inequality from 0 to $t$ we obtain

$$
-\varepsilon T \mathrm{e}^{-a^{2} \xi^{2}} \leq \mathrm{e}^{a^{2} \xi^{2} t} v(\xi, t)-v(\xi, 0) \leq \varepsilon T \mathrm{e}^{-a^{2} \xi^{2}}
$$

From which it follows

$$
\begin{aligned}
-2 \varepsilon T \mathrm{e}^{-a^{2} \xi^{2}(t+1)} & \leq v(\xi, t)-\hat{\mu}(\xi) \mathrm{e}^{-a^{2} \xi^{2} t} \\
& \leq 2 \varepsilon T \mathrm{e}^{-a^{2} \xi^{2}(t+1)}
\end{aligned}
$$

where $v(\xi, t)=\mathcal{F}[u(x, t)]$, and $\hat{\mu}(\xi)=\mathcal{F}[\mu(x)]$. In Example 1, we have established

$\mathcal{F}\left[\mathrm{e}^{-\beta|x|^{2}}\right]=\frac{1}{(2 \beta)^{n / 2}} \mathrm{e}^{-|\xi|^{2} / 4 \beta}$. Putting $n=1$, and $t=\frac{1}{4 a^{2} \beta}$, we obtain $\mathrm{e}^{-a^{2} \xi^{2} t}=\mathcal{F}\left[\frac{1}{a \sqrt{2 t}} \mathrm{e}^{-x^{2} / 4 a^{2} t}\right]$.

Now, Using the convolution theorem, from inequality (12) one has

$$
\begin{aligned}
& -\varepsilon T \mathcal{F}\left[\frac{1}{2 a \sqrt{(t+1)}} \mathrm{e}^{-\frac{1}{4 a^{2}} \frac{x^{2}}{t+1}}\right] \\
& \leq \mathcal{F}[u(x, t)]-\frac{1}{2 a \sqrt{\pi t}} \mathcal{F}\left[\mu(x) * \mathrm{e}^{-x^{2} / 4 a^{2} t}\right] \\
& \leq \varepsilon T \mathcal{F}\left[\frac{1}{2 a \sqrt{(t+1)}} \mathrm{e}^{-\frac{1}{4 a^{2}} \frac{x^{2}}{t+1}}\right]
\end{aligned}
$$

Applying inverse Fourier transform to the last inequality and using convolution theorem we have

$$
\begin{aligned}
& -\frac{\varepsilon T}{2 a \sqrt{(t+1)}} \mathrm{e}^{-\frac{1}{4 a^{2}} \frac{x^{2}}{t+1}} \\
& \leq u(x, t)-\frac{1}{2 a \sqrt{\pi t}} \int_{-\infty}^{\infty} \mu(\lambda) \mathrm{e}^{-(x-\lambda)^{2} / 4 a^{2} t} \mathrm{~d} \lambda \\
& \leq \frac{\varepsilon T}{2 a \sqrt{(t+1)}} \mathrm{e}^{-\frac{1}{4 a^{2}} \frac{x^{2}}{t+1}}
\end{aligned}
$$

Let us take

$$
w(x, t)=\frac{1}{2 a \sqrt{\pi t}} \int_{-\infty}^{\infty} \mu(\lambda) \mathrm{e}^{-(x-\lambda)^{2} / 4 a^{2} t} \mathrm{~d} \lambda .
$$

Applying arguments shown above to initial-value problem (1), (2), one can show that (13) is an exact solution of Equation (1).

To show that $w(x, 0)=\mu(x)$, we put $\mu=\frac{x-\lambda}{2 a \sqrt{t}}$. Then $\lambda=x-2 a \sqrt{t} \mu, \mathrm{d} \lambda=-2 a \sqrt{t} \mathrm{~d} \mu$, so that

$$
w(x, t)=\frac{1}{\sqrt{\pi}} \int_{-\infty}^{\infty} \mu(x-2 a \sqrt{t} \mu) \mathrm{e}^{-\mu^{2}} \mathrm{~d} \mu
$$

Hence, as $t \rightarrow 0+$ we find

$$
w(x, 0)=\frac{1}{\sqrt{\pi}} \int_{-\infty}^{\infty} \mu(x) \mathrm{e}^{-\mu^{2}} \mathrm{~d} \mu=\mu(x)
$$

Therefore the initial value problem (1), (2) is stable in the sense of Hyers-Ulam-Rassias.

More generally, the following Theorem was established for the Hyers-Ulam-Rassias stability of heat equation in $\mathbb{R}^{n}$.

Theorem 3 If $u(x, t) \in C_{1}^{2}\left(\mathbb{R}^{n} \times(0, T]\right), 0<T<\infty$, then the initial value problem (3), (4) is stable in the sense of Hyers-Ulam-Rassias.

Proof. Let $\varepsilon>0$ and $u(x, t)$ be an approximate solution of the initial value problem (3), (4). We will show that there exists a function $w(x, t) \in C_{1}^{2}\left(\mathbb{R}^{n} \times(0, T]\right)$ satisfying the Equation (3) and the initial condition (4) such that

$$
|u(x, t)-w(x, t)| \leq K \varepsilon \varphi(x, t)
$$


Taking $\varphi(x, t)=\frac{1}{(2 a \sqrt{\pi(t+1)})^{n}} \mathrm{e}^{-\frac{1}{\left.4 a^{2} t\right|^{2}}}$ then from the inequality (5), we have

$$
\begin{aligned}
& -\frac{\varepsilon}{(2 a \sqrt{(t+1)})^{n}} \mathrm{e}^{-\frac{1}{4 a^{2}} \frac{|x|^{2}}{t+1}} \\
& \leq u_{t}-a^{2} \Delta u \leq \frac{\varepsilon}{2 a \sqrt{(t+1)}} \mathrm{e}^{-\frac{1}{4 a^{2}} \frac{|x|^{2}}{t+1}}, \\
& t>0, x \in \mathbb{R}^{n}
\end{aligned}
$$

Applying Fourier Transform to inequality (14), we get

$$
\left.\left|\frac{\mathrm{d} v(\xi, t)}{\mathrm{d} t}+a^{2}\right| \xi\right|^{2} v(\xi, t) \mid \leq \varepsilon \mathrm{e}^{-a^{2}|\xi|^{2}(t+1)}
$$

Or, equivalently

$$
\begin{aligned}
-\varepsilon \mathrm{e}^{-a^{2}|\xi|^{2}} & \leq \mathrm{e}^{a^{2}|\xi|^{2} t} \frac{\mathrm{d} v(\xi, t)}{\mathrm{d} t}+a^{2}|\xi|^{2} v(\xi, t) \mathrm{e}^{a^{2}|\xi|^{2} t} \\
& \leq \varepsilon \mathrm{e}^{-a^{2}|\xi|^{2}}
\end{aligned}
$$

Integrating the inequality from 0 to $t$ we obtain

$$
-\varepsilon t \mathrm{e}^{-a^{2}|\xi|^{2}} \leq \mathrm{e}^{a^{2}|\xi|^{2} t} v(\xi, t)-v(\xi, 0) \leq \varepsilon t \mathrm{e}^{-a^{2}|\xi|^{2}}
$$

From which it follows

$$
\begin{aligned}
-\varepsilon T \mathrm{e}^{-a^{2}|\xi|^{2}(t+1)} & \leq v(\xi, t)-\hat{\mu}(\xi) \mathrm{e}^{-a^{2}|\xi|^{2} t} \\
& \leq \varepsilon T \mathrm{e}^{-a^{2}|\xi|^{2}(t+1)}
\end{aligned}
$$

where $v(\xi, t)=\mathcal{F}[u(x, t)]$, and $\hat{\mu}(\xi)=\mathcal{F}[\mu(x)]$.

Using Example 1, we find that

$$
e^{-a^{2}|\xi|^{2} t}=\mathcal{F}\left[\frac{1}{(a \sqrt{2 t})^{n}} \mathrm{e}^{-|x|^{2} / 4 a^{2} t}\right],
$$

and applying the convolution theorem, from inequality (15) one has

$$
\begin{aligned}
& -\varepsilon T \mathcal{F}\left[\frac{1}{(2 a \sqrt{(t+1)})^{n}} \mathrm{e}^{-\frac{1}{4 a^{2}} \frac{|x|^{2}}{t+1}}\right] \\
& \leq \mathcal{F}[u(x, t)]-\frac{1}{(2 a \sqrt{\pi t})^{n}} \mathcal{F}\left[\mu(x) * \mathrm{e}^{-\left|x^{2}\right| / 4 a^{2} t}\right] \\
& \leq \varepsilon T \mathcal{F}\left[\frac{1}{(2 a \sqrt{(t+1)})^{n}} \mathrm{e}^{-\frac{1}{4 a^{2}} \frac{|x|^{2}}{t+1}}\right]
\end{aligned}
$$

By applying the inverse Fourier transform to the last inequality, and then using convolution theorem we get

$$
\begin{aligned}
& -\frac{\varepsilon T}{(2 a \sqrt{t+1})^{n}} \mathrm{e}^{-\frac{1|x|^{2}}{4 a^{2} t+1}} \\
& \leq u(x, t)-\frac{1}{(2 a \sqrt{\pi t})^{n}} \int_{\mathbb{R}^{n}} \mu(\lambda) \mathrm{e}^{-|x-\lambda|^{2} / 4 a^{2} t} \mathrm{~d} \lambda \\
& \leq \frac{\varepsilon T}{(2 a \sqrt{t+1})^{n}} \mathrm{e}^{-\frac{1}{4 a^{2}} \frac{|x|^{2}}{t+1}}
\end{aligned}
$$

Now, let us take

$$
w(x, t)=\frac{1}{(2 a \sqrt{\pi t})^{n}} \int_{\mathbb{R}^{n}} \mu(\lambda) \mathrm{e}^{-|x-\lambda|^{2} / 4 a^{2} t} \mathrm{~d} \lambda .
$$

One can find that (16) is a solution of Equation (3).

To show that $w(x, 0)=\mu(x)$, we put $\mu=\frac{x-\lambda}{2 a \sqrt{t}}$. Then $\lambda=x-2 a \sqrt{t} \mu, \mathrm{d} \lambda=-2 a \sqrt{t} \mathrm{~d} \mu$, so that

$$
w(x, t)=\frac{1}{\pi^{n / 2}} \int_{\mathbb{R}^{n}} \mu(x-2 a \sqrt{t} \mu) \mathrm{e}^{-|\mu|^{2}} \mathrm{~d} \mu
$$

Hence as $t \rightarrow 0+$ we obtain

$$
w(x, 0)=\frac{1}{\pi^{n / 2}} \int_{\mathbb{R}^{n}} \mu(x) \mathrm{e}^{-|\mu|^{2}} \mathrm{~d} \mu=\mu(x)
$$

since $\frac{1}{\pi^{n / 2}} \int_{\mathbb{R}^{n}} \mathrm{e}^{-|\mu|^{2}} \mathrm{~d} \mu=1$.

Hence the initial value problem (3), (4) is stable in the sense of Hyers-Ulam-Rassias.

Theorem 4 Suppose that $u(x, t) \in C_{1}^{2}(\mathbb{R} \times(0, \infty))$ satisfies the inequality (5) with the initial condition $u(x, 0)=\mu(x)$. Then the the initial-value problem (1), (2) is stable in the sense of HURG.

Proof. Indeed, if we take $\varphi(x, t)=\frac{\mathrm{e}^{-t}}{2 a \sqrt{t+1}} \mathrm{e}^{-\frac{1}{4 a^{2}} \frac{x^{2}}{t+1}}$ then from the inequality (5), we have

$$
\begin{aligned}
& \frac{-\varepsilon \mathrm{e}^{-t}}{2 a \sqrt{t+1}} \mathrm{e}^{-\frac{1}{4 a^{2}} \frac{x^{2}}{t+1}} \\
& \leq \frac{\partial u}{\partial t}-a^{2} \frac{\partial^{2} u}{\partial x^{2}} \leq \frac{\varepsilon \mathrm{e}^{-t}}{2 a \sqrt{t+1}} \mathrm{e}^{-\frac{1}{4 a^{2}} \frac{x^{2}}{t+1}}
\end{aligned}
$$

Applying Fourier Transform to inequality (17), we get

$$
\begin{aligned}
-\varepsilon e^{-t} \mathrm{e}^{-a^{2} \xi^{2}(t+1)} & \leq \frac{\mathrm{d} v(\xi, t)}{\mathrm{d} t}+a^{2} \xi^{2} v(\xi, t) \\
& \leq \varepsilon e^{-t} \mathrm{e}^{-a^{2} \xi^{2}(t+1)}
\end{aligned}
$$

Now, by applying the same argument used above, we obtain 


$$
\begin{aligned}
& -\frac{\varepsilon\left(1-\mathrm{e}^{-t}\right)}{2 a \sqrt{t+1}} \mathrm{e}^{-\frac{1}{4 a^{2}} \frac{x^{2}}{t+1}} \\
& \leq u(x, t)-\frac{1}{2 a \sqrt{\pi t}} \int_{-\infty}^{\infty} \mu(\lambda) \mathrm{e}^{-(x-\lambda)^{2} / 4 a^{2} t} \mathrm{~d} \lambda \\
& \leq \frac{\varepsilon\left(1-\mathrm{e}^{-t}\right)}{2 a \sqrt{t+1}} \mathrm{e}^{-\frac{1}{4 a^{2}} \frac{x^{2}}{t+1}}
\end{aligned}
$$

One takes

$$
w(x, t)=\frac{1}{2 a \sqrt{\pi t}} \int_{-\infty}^{\infty} \mu(\lambda) \mathrm{e}^{-(x-\lambda)^{2} / 4 a^{2} t} \mathrm{~d} \lambda .
$$

as a solution of initial-value problem (1), (2).

Therefore the initial value problem (1), (2) is stable in the sense of HURG.

Corollary 1 Suppose that $u(x, t) \in C_{1}^{2}(\mathbb{R} \times(0, \infty))$ satisfies the inequality (5) with the initial condition (2). Then the the initial-value problem (1), (2) is asymptotically stable in the sense of Hyers-Ulam-Rassias.

Proof. It follows from Theorem 4, and letting $t \rightarrow \infty$, in (18), we infer that $\lim (u(x, t)-w(x, t))=0$.

Remark Using simitlar arguments it can be shown that the initial-value problem (3), (4) is asymptotically stable in the sense of HURG.

Example 2 We find the solution of the Cauchy problem

$$
\begin{aligned}
& 4 u_{t}=\Delta u \quad t>0 \quad x \in \mathbb{R}^{n} \\
& u(x, 0)=\mathrm{e}^{-|x|^{2} / 2}, \quad x \in \mathbb{R}^{n}
\end{aligned}
$$

Applying the same argument used in the proof of the Theorem 4 to the inequality

$$
-\frac{\varepsilon}{(\pi(t+1))^{n / 2}} \mathrm{e}^{-\frac{|x|^{2}}{t+1}} \leq u_{t}-\frac{1}{4} \Delta u \leq \frac{\varepsilon}{(\pi(t+1))^{n / 2}} \mathrm{e}^{-\frac{|x|^{2}}{t+1}}
$$

we get

$$
\begin{aligned}
& \left|u(x, t)-\frac{1}{(\sqrt{\pi t})^{n}} \int_{\mathbb{R}^{n}} \mathrm{e}^{-|x|^{2} / 2} \mathrm{e}^{-|x-\lambda|^{2} / t} \mathrm{~d} \lambda\right| \\
& \leq \frac{\varepsilon \mathrm{e}^{-t}}{(\pi(t+1))^{n / 2}} \mathrm{e}^{-\frac{|x|^{2}}{t+1}}
\end{aligned}
$$

One can show that the function

$$
w(x, t)=\frac{1}{(\pi t)^{n / 2}} \int_{\mathbb{R}^{n}} \mathrm{e}^{-|x|^{2} / 2} \mathrm{e}^{-|x-\lambda|^{2} / t} \mathrm{~d} \lambda .
$$

is a solution of the problem (19), (20).

Or, equivalently

$$
w(x, t)=\prod_{k=1}^{n} \frac{1}{(\pi t)^{1 / 2}} \int_{-\infty}^{\infty} \mathrm{e}^{-x_{k}^{2} / 2} \mathrm{e}^{-\left(x_{k}-\lambda_{k}\right)^{2} / t} \mathrm{~d} t
$$

Now, using the change of variables

$$
\begin{gathered}
z_{k}=\left(\frac{1+2 t}{2 t}\right)^{n / 2}\left(\lambda_{k}-\frac{x_{k}}{1+2 t}\right) \text { in the integral } \\
I\left(x_{k}\right)=\frac{1}{(\pi t)^{1 / 2}} \int_{-\infty}^{\infty} \mathrm{e}^{-x_{k}^{2} / 2} \mathrm{e}^{-\left(x_{k}-\lambda_{k}\right)^{2} / t} \mathrm{~d} t
\end{gathered}
$$

we obtain the integral

$$
I\left(x_{k}\right)=\frac{1}{(1+2 t)^{1 / 2}} \int_{-\infty}^{\infty} \mathrm{e}^{-x_{k}^{2} / 2(1+2 t)} \mathrm{d} \lambda_{k}, t>0
$$

Therefore we have

$$
w(x, t)=\frac{\mathrm{e}^{-|x|^{2} / 2(1+2 t)}}{(1+2 t)^{n / 2}}
$$

It is clear that $w(x, 0)=\mathrm{e}^{-|x|^{2} / 2}$.

Hence, from (21) and (23) we get

$$
\left|u(x, t)-\frac{\mathrm{e}^{-|x|^{2} / 2(1+2 t)}}{(1+2 t)^{n / 2}}\right| \leq \frac{\varepsilon \mathrm{e}^{-t}}{(\pi(t+1))^{n / 2}} \mathrm{e}^{-\frac{|x|^{2}}{t+1}}
$$

Hence the initial value problem (19), (20) is stable in the sense of HURG. Moreover, since

$$
\lim _{t \rightarrow \infty}\left(u(x, t)-\frac{\mathrm{e}^{-|x|^{2} / 2(1+2 t)}}{(1+2 t)^{n / 2}}\right)=0 \text {, then problem (19), }
$$

is asymptotically stable in the sense of HURG.

\section{A Modified Hyers-Ulam-Rassias Stability for Problem of Heat Propagation in a Finite Rod}

In this section we show how Laplace transform method can be used to esatblish the Hyers-Ulam-Rassias-Gavruta (HURG) stability of solution for heat equation

$$
\frac{\partial u}{\partial t}=a^{2} \frac{\partial^{2} u}{\partial x^{2}} \quad t>0,0<x<l
$$

with the initial condition

$$
u(x, 0)=\mu(x), 0 \leq x \leq l
$$

and the boundary conditions

$$
u(0, t)=v_{1}(t), u_{x}(0, t)=v_{2}(t), t \geq 0
$$

where $\mu(x) \in C(-\infty, \infty)$, and

$$
u(x, t) \in C_{1}^{2}(\mathbb{R} \times(0, \infty)) .
$$

We introduce the notation

$$
\mathcal{L}[u(x, t)]=U(x, p),
$$


where $\mathcal{L}[u(x, t)]=\int_{0}^{\infty} u(x, t) \mathrm{e}^{-p t} \mathrm{~d} t$.

Theorem 5 If $u(x, t) \in C_{1}^{2}(\mathbb{R} \times(0, \infty))$, then the initial-boundary value problem (24-26) is stable in the sense of Hyers-Ulam-Rassias.

Proof. Given $\varepsilon>0$, Suppose $u(x, t)$ is an approximate solution of the initial value problem (24)-(26). We show that there exists an exact solution

$w(x, t) \in C_{1}^{2}(\mathbb{R} \times(0, \infty))$ satisfying the Equation (24) such that

$$
|u(x, t)-w(x, t)| \leq K \varepsilon
$$

where $k$ is a constant that does not explicitly depend on $\varepsilon$ nor on $u(x, t)$.

From the definition of Hyers-Ulam stability we have

$$
-\varepsilon \alpha\left(t-\frac{p l^{2}}{a^{2}}\right) \leq \frac{\partial u}{\partial t}-a^{2} \frac{\partial^{2} u}{\partial x^{2}} \leq \varepsilon \alpha\left(t-\frac{l^{2}}{a^{2}}\right)
$$

where $\alpha(t-c)=0$, for $t<c$ and $\alpha(t-c)=1$, for $t>$ $c, c \geq 0$.

By applying the Laplace transform to (26), (27) we obtain

$$
\left|\mathcal{L}\left[\frac{\partial u}{\partial t}\right]-a^{2} \mathcal{L}\left[\frac{\partial^{2} u}{\partial x^{2}}\right]\right| \leq \varepsilon \mathcal{L}\left[\alpha\left(t-\frac{l^{2}}{a^{2}}\right)\right]
$$

and

$$
\begin{aligned}
& \mathcal{L}\left[u_{x}(0, t)\right]=U_{x}(0, p)=N_{1}(P), \\
& \mathcal{L}[u(0, t)]=U(0, p)=N_{2}(P),
\end{aligned}
$$

Assuming the operation of differentiation with respect to $x$ is interchangeable with integration with respect to $t$ in Laplace transform, we will get

$$
\begin{aligned}
\mathcal{L}\left[\frac{\partial^{2} u}{\partial x^{2}}\right] & =\int_{0}^{\infty} \frac{\partial^{2} u}{\partial x^{2}} \mathrm{e}^{-p t} \mathrm{~d} t=\frac{\partial^{2}}{\partial x^{2}}\left(\int_{0}^{\infty} u(x, t) \mathrm{e}^{-p t} \mathrm{~d} t\right) \\
& =\frac{\mathrm{d}^{2} U(x, p)}{\mathrm{d} x^{2}}
\end{aligned}
$$

$$
\begin{aligned}
\Delta & \leq\left|U(x, p)-N_{1}(P) x-N_{2}(P)-\frac{p}{a^{2}} \int_{0}^{x} U(s, p)(x-s) \mathrm{d} s+\frac{1}{a^{2}} \int_{0}^{x} \mu(s)(x-s) \mathrm{d} s\right|+\frac{p}{a^{2}} \int_{0}^{x}|[U(s, p)-W(s, p)](x-s)| \mathrm{d} s \\
& \leq \frac{\varepsilon l^{2}}{p a^{2}} \exp \left(-\frac{p l^{2}}{a^{2}}\right)+\frac{p}{a^{2}} \int_{0}^{x}|[U(s, p)-W(s, p)](x-s)| \mathrm{d} s
\end{aligned}
$$

with boundary condition (32).

Now consider the difference $\Delta=|U(x, p)-W(x, p)|$

Using Gronwall's inequality, we get the estimation

Or, equivalently

$$
|U(x, p)-W(x, p)| \leq \frac{\varepsilon l^{2}}{p a^{2}} \exp \left(-\frac{p l^{2}}{2 a^{2}}\right)
$$

$$
-\mathcal{L}\left\{\alpha\left(t-\frac{l^{2}}{2 a^{2}}\right)\right\} \frac{\varepsilon l^{2}}{a^{2}} \leq \mathcal{L}\{[u(x, t)]-[w(x, t)]\} \leq \frac{\varepsilon l^{2}}{a^{2}} \mathcal{L}\left\{\alpha\left(t-\frac{l^{2}}{2 a^{2}}\right)\right\}
$$


Consequently, we have

$$
\max _{0 \leq x \leq l}|u(x, t)-w(x, t)| \leq \frac{\varepsilon l^{2}}{a^{2}} \alpha\left(t-\frac{l^{2}}{2 a^{2}}\right)
$$

Hence the initial-boundary value problem (24)-(26) is stable in the sense of HURG.

Example 3 Consider the problem

$$
\begin{gathered}
\frac{\partial u}{\partial t}=4 \frac{\partial^{2} u}{\partial x^{2}} \\
t>0,0<x<4
\end{gathered}
$$

with the initial condition

$$
u(x, 0)=\cos x, 0 \leq x \leq 4
$$

with the boundary conditions

$$
u(0, t)=0, u_{x}(0, t)=0, t \geq 0
$$

By the definition of HURG stability we have

$$
-\varepsilon \alpha(t-4) \leq \frac{\partial u}{\partial t}-4 \frac{\partial^{2} u}{\partial x^{2}} \leq \varepsilon \alpha(t-4)
$$

By applying the Laplace transform to ( 36) we obtain

$$
\begin{aligned}
-\frac{\varepsilon}{p} \exp (-4 p) & \leq \frac{\mathrm{d}^{2} U}{\mathrm{~d} x^{2}}-\frac{p}{4} U+\frac{\cos X}{4} \\
& \leq \frac{\varepsilon}{4 p} \exp (-4 p)
\end{aligned}
$$

Integrating twice inequality (37) from 0 to $x$, we have

$$
\begin{aligned}
& \left|U(x, p)-\frac{p}{4} \int_{0}^{x} U(s, p)(x-s) \mathrm{d} s-\frac{\cos x}{4}\right| \\
& \leq \frac{4 \varepsilon}{p} \exp (-4 p)
\end{aligned}
$$

with the boundary conditions

$$
U(0, p)=0, U_{x}(0, p)=0
$$

It is easily to verify that the function

$$
W(x, p)=\frac{p}{4} \int_{0}^{x} W(s, p)(x-s) \mathrm{d} s+\frac{\cos x}{4}
$$

satisfies the boundary value problem

$$
\begin{gathered}
\frac{\mathrm{d}^{2} U}{\mathrm{~d} x^{2}}-\frac{p}{4} U+\frac{\cos x}{4}=0 \\
U(0, p)=0, U_{x}(0, p)=0
\end{gathered}
$$

Now consider the difference

$$
|U(x, p)-W(x, p)|
$$

$$
\begin{aligned}
\leq & \left|U(x, p)-\frac{p}{4} \int_{0}^{x} U(s, p)(x-s) \mathrm{d} s-\frac{\cos x}{4}\right| \\
& +\frac{p}{4} \int_{0}^{x}|[U(s, p)-W(s, p)](x-s)| \mathrm{d} s \\
\leq & \frac{4 \varepsilon}{p} \exp (-4 p)+\frac{p}{4} \int_{0}^{x}|[U(s, p)-W(s, p)](x-s)| \mathrm{d} s
\end{aligned}
$$

Hence, we get the estimation

$$
|U(x, p)-W(x, p)| \leq \frac{4 \varepsilon}{p} \exp (-2 p)
$$

Or, equivalently

$$
\begin{aligned}
-4 \mathcal{E} \mathcal{L}\{\alpha(t-2)\} & \leq \mathcal{L}\{[u(x, t)]-[w(x, t)]\} \\
& \leq 4 \varepsilon \mathcal{L}\{\alpha(t-2)\}
\end{aligned}
$$

Consequently, we have

$$
\max _{0 \leq x \leq l}|u(x, t)-w(x, t)| \leq 4 \varepsilon \alpha(t-2)
$$

Hence the initial-boundary value problem (33)-(35) is stable in the sense of HURG.

\section{REFERENCES}

[1] D. H. Hyers, "On the Stability of the Linear Functional Equation,” Proceedings of the National Academy of Sciences of the United States of America, Vol. 27, No. 4, 1941, pp. 222-224. doi:10.1073/pnas.27.4.222

[2] T. M. Rassias, "On the Stability of the Linear Mapping in Banach Spaces," Proceedings of the American Mathematical Society, Vol. 72, No. 2, 1978, pp. 297-300. doi:10.1090/S0002-9939-1978-0507327-1

[3] P. Gavruta, "A Generalization of the Hyers-Ulam-Rassias Stability of Approximately Additive Mappings,” Journal of Mathematical Analysis and Applications, Vol. 184, No. 3, 1994, pp. 431-436. doi:10.1006/jmaa.1994.1211

[4] S. M. Jung, "On the Hyers-Ulam-Rassias Stability of Approximately Additive Mappings,” Journal of Mathematical Analysis and Applications, Vol. 204, No. 1, 1996, pp. 221-226. doi:10.1006/jmaa.1996.0433

[5] S. M. Jung, "Hyers-Ulam-Rassias Stability of Functional Equations in Mathematical Analysis,” Hadronic Press, Palm Harbor, 2001.

[6] T. Miura, S.-E. Takahasi and H. Choda, "On the HyersUlam Stability of Real Continuous Function Valued Differentiable Map,” Tokyo Journal of Mathematics, Vol. 24, No. 2, 2001, pp. 467-476. doi:10.3836/tjm/1255958187

[7] C. G. Park, "On the Stability of the Linear Mapping in Banach Modules,” Journal of Mathematical Analysis and Applications, Vol. 275, 2002, pp. 711-720. doi:10.1016/S0022-247X(02)00386-4

[8] C. G. Park, "Homomorphisms between Poisson JC*-Algebras," Bulletin of the Brazilian Mathematical Society, Vol. 36, No. 1, 2005, pp. 79-97. 


\section{doi:10.1007/s00574-005-0029-Z}

[9] C. G. Park, Y.-S. Cho and M. Han, "Functional Inequalities Associated with Jordan-Von Neumanntype Additive Functional Equations," Journal of Inequalities and Applications, Vol. 2007, 2007, Article ID: 41820.

[10] K.-W. Jun and Y.-H. Lee, “A Generalization of the HyersUlam-Rassias Stability of the Pexiderized Quadratice Quations," Journal of Mathematical Analysis and Applications, Vol. 297, No. 1, 2004, pp. 70-86. doi:10.1016/j.jmaa.2004.04.009

[11] C. Alsina and R. Ger, "On Some Inequalities and Stability Results Related to the Exponential Function,” Journal of Inequalities and Applications, Vol. 2, No. 4, 1998, pp. 373-380.

[12] E. Takahasi, T. Miura and S. Miyajima, "On the HyersUlam Stability of the Banach Space-Valued Differential Equation $y^{\prime}=\lambda y$," Bulletin of the Korean Mathematical Society, Vol. 39, No. 2, 2002, pp. 309-315. doi:10.4134/BKMS.2002.39.2.309

[13] T. Miura, S. Miyajima and S.-E. Takahasi, "A Characterization of Hyers-Ulam Stability of First Order Linear Differential Operators," Journal of Mathematical Analysis and Applications, Vol. 286, No. 1, 2007, pp. 136-146. doi:10.1016/S0022-247X(03)00458-X

[14] S. M. Jung, "Hyers-Ulam Stability of Linear Differential Equations of First Order," Journal of Mathematical Analysis and Applications, Vol. 311, No. 1, 2005, pp. 139146. doi:10.1016/j.jmaa.2005.02.025

[15] G. Wang, M. Zhou and L. Sun, "Hyers-Ulam Stability of
Linear Differential Equations of First Order,” Applied Mathematics Letters, Vol. 21, No. 10, 2008, pp. 10241028. doi:10.1016/j.aml.2007.10.020

[16] Y. Li, "Hyers-Ulam Stability of Linear Differential Equations,” Thai Journal of Mathematics, Vol. 8, No. 2, 2010, pp. 215-219.

[17] Y. Li and Y. Shen, "Hyers-Ulam Stability of Nonhomogeneous Linear Differential Equations of Second Order, Internat,” Journal of Mathematical Sciences, Vol. 2009, 2009, Article ID: 576852.

[18] P. Gavruta, S. Jung and Y. Li, "Hyers-Ulam Stability for Second-Order Linear Differential Equations with Boundary Conditions,” Electron Journal of Differential Equations, Vol. 2011, No. 80, 2011, pp. 1-7.

[19] S. M. Jung, "Hyers-Ulam Stability of Linear Partial Differential Equations of First Order," Applied Mathematics Letters, Vol. 22, No. 1, 2009, pp. 70-74. doi:10.1016/j.aml.2008.02.006

[20] M. E. Gordji, Y. J. Cho, M. B. Ghaemi and B. Alizadeh, "Stability of the Exact Second Order Partial Differential Equations,” Journal of Inequalities and Applications, Vol. 2011, 2011, Article ID: 306275. doi:10.1186/1029-242X-2011-81

[21] N. Lungu and C. Craciun, "Ulam-Hyers-Rassias Stability of a Hyperbolic Partial Differential Equation,” ISRN Mathematical Analysis, Vol. 2012, 2012, Article ID: 609754.

[22] L. C. Evans, "Partial Differential Equations," 2nd Edition, Graduate Studies in Mathematics, V19 AMS, 2010. 